\title{
Infrared MnI laboratory oscillator strengths for the study of late type stars and ultracool dwarfs
}

\author{
R. Blackwell-Whitehead ${ }^{1,2}$, Y. V. Pavlenko ${ }^{3,4}$, G. Nave ${ }^{5}$, J. C. Pickering ${ }^{2}$, H. R. A. Jones ${ }^{3}$, \\ Y. Lyubchik ${ }^{4}$, and H. Nilsson ${ }^{1}$
}

\author{
${ }^{1}$ Lund Observatory, Box 43, 22100 Lund, Sweden \\ e-mail: r.blackwell@astro.lu.se \\ 2 Blackett Laboratory, Imperial College London, London SW7 2AZ, UK \\ 3 Centre for Astrophysics Research, University of Hertfordshire, College Lane, Hatfield, Hertfordshire AL10 9AB, UK \\ ${ }^{4}$ Main Astronomical Observatory of the Academy of Sciences of Ukraine, Zabolotnoho 27, Kyiv 03680, Ukraine \\ 5 Atomic Physics Division, National Institute of Standards and Technology, 100 Bureau Dr., Gaithersburg, MD 20899, USA
}

Received 17 May 2010 / Accepted 3 September 2010

\begin{abstract}
Aims. The aim of our new laboratory measurements is to measure accurate absolute oscillator strengths for neutral manganese transitions in the infrared needed for the study of late-type stars and ultracool dwarfs.

Methods. Branching fractions have been measured by high resolution Fourier transform spectroscopy and combined with radiative level lifetimes in the literature to yield oscillator strengths.

Results. We present experimental oscillator strengths for $20 \mathrm{Mn}$ I transitions in the wavelength range 3216 to $13997 \AA$, 15 of which are in the infrared. The transitions at $12899 \AA$ and $12975 \AA$ are observed as strong features in the spectra of late-type stars and ultracool dwarfs. We have fitted our calculated spectra to the observed Mn I lines in spectra of late-type stars. Using the new experimentally measured $\mathrm{MnI} \log (g f)$ values together with existing data for Mn I hyperfine structure splitting factors we determined the manganese abundance to be $\log N(\mathrm{Mn})=-6.65 \pm 0.05$ in the atmosphere of the Sun, $\log N(\mathrm{Mn})=6.95 \pm 0.20$ in the atmosphere of Arcturus, and $\log N(\mathrm{Mn})=-6.70 \pm 0.20$ in the atmosphere of M 9.5 dwarf 2MASSW $0140026+270150$.
\end{abstract}

Key words. atomic data - line: identification - methods: laboratory - stars: late-type

\section{Introduction}

Manganese is an iron-peak element and the nucleosynthesis path that leads to its formation is relatively well understood. However, it remains unclear which objects are the main donors of manganese to the Galaxy at different times of its evolution. Nevertheless, manganese is widely used for the investigation of the chemical evolution of the disk and halo of our Galaxy (see Sobeck et al. 2006, and references therein).

Accurate atomic oscillator strengths $(f$-values and $\log (g f))$ are required for the correct interpretation of the physical properties and processes in stellar and sub-stellar objects. In particular, oscillator strengths for atomic lines in the infrared (IR) spectral region are needed for the study of the spectra of late-type stars, ultracool dwarfs, and dust obscured objects such as young stars and the centre of galaxies. Accurate oscillator strengths are of particular importance to the study of late type stars and ultracool dwarfs where the spectral energy distribution peaks in the IR and is dominated by spectral lines from neutral atoms and molecules (see Lyubchik et al. 2004; Jones et al. 2005).

Over the past ten years there has been an increase in IR spectral observations of astrophysical objects with the advent of new IR spectrographs on ground based and satellite borne telescopes. However, there are only a relatively small number of experimentally measured oscillator strengths for IR spectral lines compared to the number of measured oscillator strengths for visible spectral lines available in the literature. The status of oscillator strengths in the atomic database has been discussed by
Wahlgren \& Johansson (2003), Johansson (2005), Brickhouse et al. (2006) and Blackwell-Whitehead et al. (2008), and there have been calls for more IR measurements including Lyubchik et al. (2004), and Bigot \& Thévenin (2006).

The current laboratory atomic database for Mn I oscillator strengths is dominated by transitions in the UV and visible. Our previous oscillator strength measurements for Mn I, BlackwellWhitehead et al. (2005a), include 44 transitions from 2090 to $27800 \AA$ of which six transitions are in the IR. Only eleven experimentally measured oscillator strengths for Mn I have been published for $\lambda>6520 \AA$, see Blackwell-Whitehead et al. (2005a). Booth et al. (1984) reported $58 \mathrm{Mn} \mathrm{I} f$-values including the UV and visible resonance transitions from the $3 d^{5}\left({ }^{6} \mathrm{~S}\right) 4 \mathrm{~s} 4 \mathrm{p}$ $z^{4} \mathrm{P}_{J}^{\circ}$ and $3 \mathrm{~d}^{5}\left({ }^{6} \mathrm{~S}\right) 4 \mathrm{~s} 4 \mathrm{p} z{ }^{6} \mathrm{P}_{J}^{\circ}$ levels. However, no $f$-values have been published for the IR transitions from the $z{ }^{4} \mathrm{P}_{J}^{\circ}$ and $z{ }^{6} \mathrm{P}_{J}^{\circ}$ levels despite these transitions being amongst the strongest Mn I transitions for $10000<\lambda<15000 \AA$. In our current work we measured Mn I oscillator strengths for transitions from the $z^{4} \mathrm{P}_{J}^{\circ}$ and $z{ }^{6} \mathrm{P}_{J}^{\circ}$ upper levels by combining branching fractions $(B F \mathrm{~s})$ measured by high resolution Fourier transform spectrometry with known level lifetimes $(\tau)$. These include the $12899 \AA$ and $12875 \AA$ spectral lines that are observed as strong features in the spectra of ultracool dwarf stars, see Lyubchik et al. (2007).

\section{Laboratory measurements}

The oscillator strengths have been determined by combining $B F \mathrm{~s}$ with radiative lifetimes in the same manner as described 
in Blackwell-Whitehead et al. (2005a). The spectrum of Mn I was recorded in the UV to visible spectral range (1600 $\AA$ to $8000 \AA$ ) using the Imperial College high resolution Fourier transform spectrometer (FTS) (Pickering 2002), and in the visible to IR spectral range ( $3500 \AA$ to $55000 \AA$ ) using the $2 \mathrm{~m}$ FTS at the National Institute of Standards and Technology (NIST) (Nave et al. 1997). The light source for the NIST and Imperial College measurements was a hollow cathode lamp (HCL) with a manganese cathode using either an argon or neon buffer gas. Two manganese cathodes were used in these measurements. The NIST cathode was an alloy of $95 \%$ manganese and 5\% copper, the cathode used at Imperial College was an alloy of $88 \%$ manganese and $12 \%$ nickel. The optimal running conditions for the HCL were found to be a pressure of $340 \mathrm{~Pa}$ of neon with currents of $200 \mathrm{~mA}$ to $500 \mathrm{~mA}$. Further details of the measurements and their analysis are given in Blackwell-Whitehead et al. (2005a). The spectra were recorded at a range of currents to determine an intensity versus current curve of growth for each line to determine if any lines were self absorbed, which would lead to erroneous relative line intensities. It was found that a HCL current of $200 \mathrm{~mA}$ was used to observe absorption free spectra of the UV and visible resonance lines and a higher HCL current of $500 \mathrm{~mA}$ was used for the measurement of the relatively weaker IR transitions. In addition, the line profile of each transition was fitted using published hyperfine structure (HFS) constants for the upper and lower level of the transition (Handrich et al. 1969; Dembczyński et al. 1979; Brodzinski et al. 1987; BlackwellWhitehead et al. 2005b). The residual value of the fit was found to be the same as the background noise level indicating that no self absorption was present.

The manganese spectra were intensity calibrated using tungsten intensity standard lamps. The Imperial College tungsten intensity standard lamp was calibrated by the National Physical Laboratory, UK, and the NIST tungsten intensity standard lamp was calibrated by Optronics Laboratories ${ }^{1}$. Both lamps have a minimum radiance uncertainty of 3 percent in the spectral region used for the intensity calibration. The tungsten spectra were recorded before and after the manganese spectra and compared to determine if the instrumental response had changed during measurement of the manganese spectrum. In each case, the instrument response did not vary by more than the uncertainty in the relative radiance of the tungsten lamp. The measured tungsten spectra were used to determine the instrument response, and this was used to calibrate the relative intensity of the Mn I lines. The Mn I line profiles were fitted by employing a centre of gravity fit using the XGremlin software by Nave et al. (1997). To observe all lines from the $z^{4} \mathrm{P}_{J}^{\circ}$ and $z^{6} \mathrm{P}_{J}^{\circ}$ upper levels, spectra were recorded in two overlapping spectral regions. The intensity calibration of the visible to IR region was placed on the same intensity scale as the UV to visible region using intermediate transitions in the $18000 \mathrm{~cm}^{-1}$ to $18500 \mathrm{~cm}^{-1}$ region $\left(3 \mathrm{~d}^{6}\left({ }^{5} \mathrm{D}\right) 4 \mathrm{~s}\right.$ $\left.a^{6} \mathrm{D}_{J}-3 \mathrm{~d}^{5}\left({ }^{6} \mathrm{~S}\right) 4 \mathrm{~s} 4 \mathrm{p} y{ }^{6} \mathrm{P}_{J}^{\circ}\right)$. The intermediate transitions are from upper levels with the same configuration and comparable level energy, which indicates that the $z{ }^{4} \mathrm{P}_{J}^{\circ}, z{ }^{6} \mathrm{P}_{J}^{\circ}$ and $y{ }^{6} \mathrm{P}_{J}^{\circ}$ levels have comparable level populations. Curves of growth for the intermediate lines indicated that no self absorption was present. Furthermore, an estimate of the change in level population between the $z^{4} \mathrm{P}_{J}^{\circ}, z^{6} \mathrm{P}_{J}^{\circ}$ and $y^{6} \mathrm{P}_{J}^{\circ}$ levels was measured by comparing the intensity ratios between the a ${ }^{6} \mathrm{D}_{J}-y{ }^{6} \mathrm{P}_{J}^{\circ}$ transitions and

\footnotetext{
1 Certain trade names and products are mentioned in the text in order to adequately identify the apparatus used to obtain the measurements. In no case does such identification imply recommendation or endorsement by NIST or any of the coauthor institutes.
}

transitions from the $z^{4} \mathrm{P}_{J}^{\circ}$ and $z{ }^{6} \mathrm{P}_{J}^{\circ}$ upper levels under different HCL conditions. The intensity ratios did not vary by more than the uncertainty in the measured relative line intensities, indicating that the level populations remained constant to within a few percent.

\section{Laboratory oscillator strengths}

Table 1 presents the $B F$ s, transition probabilities and oscillator strengths. The oscillator strengths were obtained by combining the $B F$ s with the published radiative lifetimes of Kronfeldt et al. (1985) and Schnabel et al. (1995). The Mn I Ritz wavenumbers in Table 1 are determined from the upper and lower energy level values from the NIST atomic Spectra Database (Ralchenko et al. 2009), which are taken from the term analysis of Catalán et al. (1964). The air wavelengths in Table 1 have been determined with the Edlén (1966) equation, and include the more recent update for the refractive index of air, Eq. (3) in Birch \& Downs (1994). All lines measured in this work have a peak signal to noise ratio of more than 100 , and the $B F$ uncertainties are dominated by the tungsten lamp calibration uncertainty and the uncertainty in the intensity calibration "cross-over" between the two spectral regions. The uncertainty in the oscillator strength is determined from the $B F$ and lifetime uncertainty using the criteria discussed by Sikström et al. (2002) and follows the NIST guidelines for evaluating and expressing uncertainty (Taylor \& Kuyatt 1994).

It can be seen that our oscillator strengths for the transitions from the $z^{6} \mathrm{P}_{J}^{\circ}$ levels agree, to within the uncertainty, with the previous UV and visible measurements by Booth et al. (1984). There is also a good agreement with our laboratory $\log (g f)$ values for the transitions from the $z{ }^{6} \mathrm{P}_{J}^{\circ}$ levels and the semiempirical calculated $\log (g f)$ values of Kurucz \& Bell (1995). The measured $\log (g f)$ values for UV transitions from the $z{ }^{4} \mathrm{P}_{J}^{\circ}$ levels agree with both Booth et al. (1984) and Kurucz \& Bell (1995) to within the uncertainties. However, the semi-empirical $\log (g f)$ values of Kurucz \& Bell (1995) for IR transitions from the $z^{4} \mathrm{P}_{J}^{\circ}$ levels are approximately $30 \%(0.12$ dex where the unit dex is $\log _{10}$ of the ratio of the two values) stronger than our values. The difference between the measured $\log (g f)$ s and the semiempirical $\log (g f)$ s is larger than the uncertainty in the measured values. It is possible that the semi-empirical calculations predict more level mixing than is present in the actual system. If the $z{ }^{4} \mathrm{P}_{J}^{\circ}$ levels have less mixing than predicted by the semiempirical calculations of Kurucz \& Bell (1995), then the measured $\log (g f)$ s will be weaker.

The effect of hyperfine splitting on the fine structure levels and on the line profiles of Mn I transitions in the IR can be seen in an example shown in Fig. 1. The hyperfine splitting increases the width and decreases the peak intensity of the line profile. Prochaska \& McWilliam (2000) discuss the importance of including hyperfine splitting in the analysis of elemental abundances in stars. In particular, if HFS is not taken into account, the chemical elemental abundance may be underestimated or it may erroneously be assumed that the line is blended with some unknown feature. To assist in the correct interpretation of the oscillator strengths in Table 1 we provide wavenumbers, wavelengths and oscillator strengths in Table 2 for the HFS component lines in the IR transitions $3 d^{6}\left({ }^{5} \mathrm{D}\right) 4 \mathrm{~s}$ a ${ }^{6} \mathrm{D}_{J}-3 \mathrm{~d}^{5}\left({ }^{6} \mathrm{~S}\right) 4 \mathrm{~s} 4 \mathrm{p}$ $z^{6} \mathrm{P}_{J}^{\circ}$ and $3 \mathrm{~d}^{6}\left({ }^{5} \mathrm{D}\right) 4 \mathrm{~s}$ a ${ }^{4} \mathrm{D}_{J}-3 \mathrm{~d}^{5}\left({ }^{6} \mathrm{~S}\right) 4 \mathrm{~s} 4 \mathrm{p} z{ }^{4} \mathrm{P}_{J}^{\circ}$.

The wavenumber of each transition from the upper hyperfine structure level to the lower hyperfine structure level, $\sigma_{\text {HFS trans }}$, 
R. Blackwell-Whitehead et al.: Infrared Mn I laboratory oscillator strengths for the study of late type stars and ultracool dwarfs

Table 1. New and remeasured laboratory oscillator strengths for Mn I.

\begin{tabular}{|c|c|c|c|c|c|c|c|c|c|c|c|}
\hline \multirow{2}{*}{$\begin{array}{l}\text { Upper } \\
\text { level }\end{array}$} & \multirow{2}{*}{$\begin{array}{l}\text { Lower } \\
\text { level }^{a}\end{array}$} & \multirow{2}{*}{$\begin{array}{c}\text { Wavenumber }{ }^{b} \\
\left(\mathrm{~cm}^{-1}\right)\end{array}$} & \multirow{2}{*}{$\begin{array}{l}\lambda_{\text {air }} \\
(\AA)\end{array}$} & \multirow[t]{2}{*}{$B F$} & \multirow{2}{*}{$\begin{array}{c}\text { BF Unc. } \\
(\%)\end{array}$} & \multirow{2}{*}{$\begin{array}{c}T P^{c} \\
\left(10^{7} \mathrm{~s}^{-1}\right)\end{array}$} & \multicolumn{2}{|c|}{ This Work } & \multicolumn{2}{|c|}{ Previous Work ${ }^{e}$} & \multirow{2}{*}{$\begin{array}{l}\text { Calc }^{f} \\
\log (g f)\end{array}$} \\
\hline & & & & & & & $\log (g f)$ & Unc. $(\operatorname{dex})^{d}$ & $\log (g f)$ & Unc. (dex) & \\
\hline $3 d^{5}\left({ }^{6} \mathrm{~S}\right) 4 \mathrm{~s} 4 \mathrm{p} z^{6} \mathrm{P}_{7 / 2}^{\circ}$ & $a{ }^{6} S_{5 / 2}$ & 24802.25 & 4030.75 & 0.965 & 4 & $16.01(0.80)$ & -0.51 & 0.02 & -0.47 & 0.08 & -0.52 \\
\hline$E=24802.25 \mathrm{~cm}^{-1}$ & $a^{6} D_{9 / 2}$ & 7749.96 & 12899.76 & 0.026 & 13 & $0.43(0.05)$ & -1.07 & 0.05 & & & -1.06 \\
\hline$\tau=60.3 \pm 1.3 \mathrm{~ns}^{g}$ & $a^{6} D_{7 / 2}$ & 7520.25 & 13293.80 & 0.007 & 13 & $0.12(0.02)$ & -1.58 & 0.05 & & & -1.61 \\
\hline Residual & $\mathrm{a}^{6} \mathrm{D}_{5 / 2}$ & 7350.73 & 13600.37 & $\begin{array}{l}0.001 \\
0.000\end{array}$ & 13 & $0.02(0.003)$ & -2.33 & 0.05 & & & -2.40 \\
\hline $3 \mathrm{~d}^{5}\left({ }^{6} \mathrm{~S}\right) 4 \mathrm{~s} 4 \mathrm{p} z^{6} \mathrm{P}_{5 / 2}^{\circ}$ & $a{ }^{6} S_{5 / 2}$ & 24788.05 & 4033.06 & 0.967 & 4 & $15.16(0.76)$ & -0.65 & 0.02 & -0.62 & 0.08 & -0.62 \\
\hline$E=24788.05 \mathrm{~cm}^{-1}$ & $a^{6} D_{7 / 2}$ & 7506.05 & 13318.94 & 0.017 & 13 & $0.27(0.03)$ & -1.37 & 0.05 & & & -1.36 \\
\hline$\tau=63.8 \pm 1.4 \mathrm{~ns}^{f}$ & $\mathrm{a}^{6} \mathrm{D}_{5 / 2}$ & 7336.53 & 13626.96 & 0.012 & 13 & $0.19(0.02)$ & -1.51 & 0.05 & & & -1.52 \\
\hline Residual & $a^{6} D_{3 / 2}$ & 7219.57 & 13847.46 & $\begin{array}{l}0.004 \\
0.000\end{array}$ & 13 & $0.06(0.01)$ & -1.98 & 0.05 & & & -2.00 \\
\hline $3 \mathrm{~d}^{5}\left({ }^{6} \mathrm{~S}\right) 4 \mathrm{~s} 4 \mathrm{p} z^{6} \mathrm{P}_{3 / 2}^{\circ}$ & $a{ }^{6} S_{5 / 2}$ & 24779.32 & 4034.48 & 0.969 & 4 & $14.66(0.73)$ & -0.84 & 0.02 & -0.81 & 0.08 & -0.81 \\
\hline$E=24779.32 \mathrm{~cm}^{-1}$ & $\mathrm{a}^{6} \mathrm{D}_{5 / 2}$ & 7327.80 & 13642.93 & 0.009 & 13 & $0.14(0.02)$ & -1.82 & 0.05 & & & -1.81 \\
\hline$\tau=66.1 \pm 1.4 \mathrm{~ns}^{f}$ & $a^{6} D_{3 / 2}$ & 7210.84 & 13864.22 & 0.013 & 13 & $0.20(0.03)$ & -1.65 & 0.05 & & & -1.64 \\
\hline Residual & $a^{6} D_{1 / 2}$ & 7142.11 & 13997.52 & $\begin{array}{l}0.009 \\
0.000\end{array}$ & 13 & $0.14(0.02)$ & -1.78 & 0.05 & & & -1.79 \\
\hline $3 d^{5}\left({ }^{6} \mathrm{~S}\right) 4 \mathrm{~s} 4 \mathrm{p} z^{4} \mathrm{P}_{5 / 2}^{\circ}$ & $a{ }^{6} S_{5 / 2}$ & 31001.15 & 3224.76 & 0.348 & 8 & $0.36(0.04)$ & -2.47 & 0.04 & -2.45 & 0.08 & -2.48 \\
\hline$E=31001.15 \mathrm{~cm}^{-1}$ & $a^{4} D_{7 / 2}$ & 7704.48 & 12975.91 & 0.524 & 6 & $0.54(0.04)$ & -1.09 & 0.03 & & & -0.94 \\
\hline$\tau=970 \pm 50 \mathrm{~ns}^{f}$ & $a^{4} D_{5 / 2}$ & 7451.95 & 13415.64 & 0.113 & 9 & $0.12(0.01)$ & -1.73 & 0.04 & & & -1.60 \\
\hline Residual & $a^{4} D_{3 / 2}$ & 7281.63 & 13729.44 & $\begin{array}{l}0.013 \\
0.002\end{array}$ & 10 & $0.01(0.001)$ & -2.65 & 0.04 & & & -2.57 \\
\hline $3 \mathrm{~d}^{5}\left({ }^{6} \mathrm{~S}\right) 4 \mathrm{~s} 4 \mathrm{p} z^{4} \mathrm{P}_{3 / 2}^{\circ}$ & $a{ }^{6} S_{5 / 2}$ & 31076.42 & 3216.95 & 0.261 & 8 & $0.23(0.02)$ & -2.84 & 0.04 & -2.82 & 0.08 & -2.82 \\
\hline$E=31076.42 \mathrm{~cm}^{3 / 2}$ & $a^{4} D_{5 / 2}$ & 7527.22 & 13281.49 & 0.473 & 6 & $0.42(0.03)$ & -1.35 & 0.03 & & & -1.23 \\
\hline$\tau=1120 \pm 50 \mathrm{~ns}^{f}$ & $a^{4} D_{3 / 2}$ & 7356.90 & 13588.97 & 0.229 & 8 & $0.21(0.02)$ & -1.64 & 0.04 & & & -1.53 \\
\hline & $a^{4} D_{1 / 2}$ & 7257.55 & 13774.99 & 0.035 & 9 & $0.03(0.003)$ & -2.45 & 0.04 & & & -2.35 \\
\hline Residual & & & & 0.002 & & & & & & & \\
\hline
\end{tabular}

Notes. ${ }^{(a)}$ Full term designations for the lower levels are $3 \mathrm{~d}^{6}\left({ }^{5} \mathrm{D}\right) 4 \mathrm{~s} \mathrm{a} \mathrm{a}^{4,6} \mathrm{D}_{J}$ and $3 \mathrm{~d}^{5}\left({ }^{6} \mathrm{~S}\right) 4 \mathrm{~s}^{2} \mathrm{a}^{6} \mathrm{~S}_{5 / 2} \cdot{ }^{(b)}$ Ritz wavenumber. ${ }^{(c)}$ Transition probability, the uncertainty is given in brackets $( \pm)$. ${ }^{(d)}$ The uncertainty in the $\log (g f)$ is expressed in dex, where \pm 0.01 dex corresponds to approximately \pm 2.5 percent. ${ }^{(e)}$ The previous laboratory $\log (g f)$ s from Booth et al. (1984), the uncertainties in the $\log (g f)$ s of Booth et al. (1984) are those assigned by NIST, Fuhr \& Wiese (2003). ${ }^{(f)}$ Semi-empirical $\log (g f)$ calculations of Kurucz \& Bell (1995). ${ }^{(g)}$ Radiative lifetime values, $\tau$, for $z^{6} \mathrm{P}_{J}^{\circ}$ are from Schnabel et al. (1995) and for $z^{4} \mathrm{P}_{J}^{\circ}$ are from Kronfeldt et al. (1985).

in Table 2 is determined from the wavenumber for the finestructure transition, $\sigma_{\mathrm{FS}}$, using:

$\sigma_{\mathrm{HFS} \text { trans }}=\sigma_{\mathrm{FS}}-\frac{K_{\mathrm{l}} A_{1}}{2}+\frac{K_{\mathrm{u}} A_{\mathrm{u}}}{2}$

where $A_{\mathrm{u}}$ and $A_{\mathrm{l}}$ are the magnetic dipole hyperfine interaction constants for the upper and lower fine structure levels; and $K$ is defined as:

$K=F(F+1)-J(J+1)-I(I+1)$

where $F$ is the quantum number associated with the total angular momentum of the electrons, $J$, and the nuclear spin, $I$. For manganese the spin of the nucleus $I=5 / 2$. Equation (1) excludes the contribution from the electric quadrupole hyperfine interaction constant $B$ which is relatively small when compared to the magnetic dipole hyperfine interaction constant $A$ for the levels considered in this paper, see Kuhn (1964).

Several of the IR transitions included in our work (12 899.8, 12 975.9, $13281.5,13319.0$, and $13642.9 \AA$ ) have been studied by Meléndez (1999) who has determined the wavelength of individual hyperfine structure components by analysing Mn I transitions in solar photospheric spectra. However, Meléndez notes that several of these lines have blended features and he does not provide HFS constants for the upper and lower levels of the transitions.

\section{Modelling Mn I lines in the spectra of the Sun, Arcturus and ultracool dwarfs}

We have computed the synthetic spectra of late-type stars and brown dwarfs using the WITA6 programme (Pavlenko 2000). The model atmospheres for the Sun (spectral classification $=\mathrm{G} 2 \mathrm{~V}, T_{\text {eff }} / \log (g)=5770 / 4.44$, and abundances from Gurtovenko \& Kostik 1989), and Arcturus (K2III, 4300/1.5, and abundances from Peterson et al. 1993) have been computed with ATLAS 12 (Kurucz 1993; Pavlenko 2003). The calculated spectra of the brown dwarf 2MASSW $0140026+270150$ were computed with the DUSTY 2000/4.5/0 model atmosphere (Allard et al. 2001). The atomic line data for species other than Mn I were taken from the Vienna Atomic Line Database (VALD) (Kupka et al. 1999). We used molecular line lists from different sources: TiO (Plez 1998), FeH (Dulick et al. 2003), $\mathrm{CrH}$ (Burrows et al. 2002) as well as $\mathrm{H}_{2} \mathrm{O}$ line list BT2 (Barber et al. 2006). For Arcturus we used the abundances of Peterson et al. (1993) and the spectra of the Sun and brown dwarfs were computed with the solar abundances reported by Anders \& Grevesse (1989). The absorption lines are hyperfine split and each hyperfine component line (Table 2) has been fitted using the Voigt function $H(a, v)$ and the formulae of Unsöld (1955) to calculate the damping constants. Theoretical spectra were computed with a wavelength step $0.01 \AA$ and convolved with Gaussians to match 
Table 2. Wavenumber and wavelength of the HFS component lines in the IR transitions $3 \mathrm{~d}^{6}\left({ }^{5} \mathrm{D}\right) 4 \mathrm{~s}$ a ${ }^{6} \mathrm{D}_{J}-3 \mathrm{~d}^{5}\left({ }^{6} \mathrm{~S}\right) 4 \mathrm{~s} 4 \mathrm{p} z^{6} \mathrm{P}_{J}^{\circ}$ and $3 \mathrm{~d}^{6}\left({ }^{5} \mathrm{D}\right) 4 \mathrm{~s}$ a ${ }^{4} D_{J}-3 d^{5}\left({ }^{6} S\right) 4 s 4 p z^{4} P_{J}^{\circ}$.

\begin{tabular}{|c|c|c|c|c|c|c|c|c|c|c|}
\hline \multirow{2}{*}{$\begin{array}{l}\text { Upper } \\
\text { level }\end{array}$} & \multirow{2}{*}{$\begin{array}{c}\text { Lower } \\
\text { level }\end{array}$} & \multicolumn{4}{|c|}{ HFS Constants $\left(\mathrm{cm}^{-1}\right)^{a}$} & \multicolumn{2}{|c|}{ HFS levels } & \multicolumn{2}{|c|}{ HFS transitions ${ }^{b}$} & \multirow{2}{*}{$\begin{array}{c}\text { HFS Comp. }^{c} \\
\quad \log (g f) \\
\end{array}$} \\
\hline & & $A_{\text {upper }}$ & $B_{\text {upper }}$ & $A_{\text {lower }}$ & $B_{\text {lower }}$ & $F_{\text {upper }}$ & $F_{\text {lower }}$ & $\sigma\left(\mathrm{cm}^{-1}\right)$ & $\lambda_{\text {air }}(\AA)$ & \\
\hline$z^{6} \mathrm{P}_{7 / 2}^{\circ}$ & $a^{6} D_{9 / 2}$ & $\begin{array}{c}0.0143 \\
(0.0001)\end{array}$ & $\begin{array}{c}0.0022 \\
(0.0001)\end{array}$ & $\begin{array}{c}0.0170 \\
(0.0003)\end{array}$ & $\begin{array}{c}0.0044 \\
(0.0040)\end{array}$ & $\begin{array}{l}6.0 \\
5.0 \\
4.0 \\
3.0 \\
2.0 \\
3.0 \\
4.0 \\
1.0 \\
5.0 \\
6.0 \\
2.0 \\
3.0 \\
4.0 \\
5.0 \\
6.0\end{array}$ & $\begin{array}{l}5.0 \\
4.0 \\
3.0 \\
2.0 \\
2.0 \\
3.0 \\
4.0 \\
2.0 \\
5.0 \\
6.0 \\
3.0 \\
4.0 \\
5.0 \\
6.0 \\
7.0\end{array}$ & $\begin{array}{l}7750.1163 \\
7750.1143 \\
7750.1099 \\
7750.1031 \\
7750.0607 \\
7750.0530 \\
7750.0427 \\
7750.0325 \\
7750.0297 \\
7750.0141 \\
7750.0106 \\
7749.9858 \\
7749.9582 \\
7749.9275 \\
7749.8937\end{array}$ & $\begin{array}{l}12899.5037 \\
12899.5071 \\
12899.5144 \\
12899.5256 \\
12899.5962 \\
12899.6090 \\
12899.6262 \\
12899.6431 \\
12899.6478 \\
12899.6738 \\
12899.6797 \\
12899.7208 \\
12899.7669 \\
12899.8179 \\
12899.8742\end{array}$ & $\begin{array}{l}-4.18 \\
-3.85 \\
-3.75 \\
-3.88 \\
-2.78 \\
-2.60 \\
-2.54 \\
-2.27 \\
-2.58 \\
-2.77 \\
-2.14 \\
-2.01 \\
-1.89 \\
-1.78 \\
-1.67\end{array}$ \\
\hline$z^{4} \mathrm{P}_{5 / 2}^{\circ}$ & $a{ }^{4} D_{7 / 2}$ & $\begin{array}{l}-0.0203 \\
(0.0001)\end{array}$ & $\begin{array}{c}0.0025 \\
(0.0005)\end{array}$ & $\begin{array}{l}-0.0054 \\
(0.0001)\end{array}$ & $\begin{array}{c}0.0000 \\
(-)\end{array}$ & $\begin{array}{l}0.0 \\
1.0 \\
1.0 \\
2.0 \\
2.0 \\
2.0 \\
3.0 \\
3.0 \\
3.0 \\
4.0 \\
4.0 \\
4.0 \\
5.0 \\
5.0 \\
5.0\end{array}$ & $\begin{array}{l}1.0 \\
2.0 \\
1.0 \\
3.0 \\
2.0 \\
1.0 \\
4.0 \\
3.0 \\
2.0 \\
5.0 \\
4.0 \\
3.0 \\
6.0 \\
5.0 \\
4.0\end{array}$ & $\begin{array}{l}7704.5977 \\
7704.5878 \\
7704.5770 \\
7704.5628 \\
7704.5466 \\
7704.5358 \\
7704.5229 \\
7704.5013 \\
7704.4851 \\
7704.4686 \\
7704.4416 \\
7704.4200 \\
7704.4005 \\
7704.3681 \\
7704.3411\end{array}$ & $\begin{array}{l}12975.7138 \\
12975.7305 \\
12975.7487 \\
12975.7727 \\
12975.7999 \\
12975.8181 \\
12975.8399 \\
12975.8762 \\
12975.9035 \\
12975.9313 \\
12975.9768 \\
12976.0132 \\
12976.0460 \\
12976.1006 \\
12976.1460\end{array}$ & $\begin{array}{l}-2.65 \\
-2.36 \\
-2.62 \\
-2.14 \\
-2.44 \\
-3.39 \\
-1.96 \\
-2.37 \\
-3.32 \\
-1.80 \\
-2.40 \\
-3.44 \\
-1.66 \\
-2.57 \\
-3.79\end{array}$ \\
\hline$z^{4} \mathrm{P}_{3 / 2}^{\circ}$ & $a^{4} D_{5 / 2}$ & $\begin{array}{l}-0.0271 \\
(0.0001)\end{array}$ & $\begin{array}{l}-0.0013 \\
(0.0010)\end{array}$ & $\begin{array}{l}-0.0046 \\
(0.0001)\end{array}$ & $\begin{array}{c}0.0000 \\
(-)\end{array}$ & $\begin{array}{l}1.0 \\
1.0 \\
1.0 \\
2.0 \\
2.0 \\
2.0 \\
3.0 \\
3.0 \\
3.0 \\
4.0 \\
4.0 \\
4.0\end{array}$ & $\begin{array}{l}2.0 \\
1.0 \\
0.0 \\
3.0 \\
2.0 \\
1.0 \\
4.0 \\
3.0 \\
2.0 \\
5.0 \\
4.0 \\
3.0\end{array}$ & $\begin{array}{l}7527.3355 \\
7527.3263 \\
7527.3217 \\
7527.2957 \\
7527.2819 \\
7527.2727 \\
7527.2332 \\
7527.2148 \\
7527.2010 \\
7527.1471 \\
7527.1241 \\
7527.1057\end{array}$ & $\begin{array}{l}13281.2812 \\
13281.2975 \\
13281.3056 \\
13281.3513 \\
13281.3756 \\
13281.3919 \\
13281.4616 \\
13281.4941 \\
13281.5184 \\
13281.6135 \\
13281.6541 \\
13281.6865\end{array}$ & $\begin{array}{l}-2.76 \\
-2.58 \\
-2.91 \\
-2.35 \\
-2.43 \\
-2.95 \\
-2.08 \\
-2.41 \\
-3.13 \\
-1.86 \\
-2.55 \\
-3.51\end{array}$ \\
\hline$z^{6} \mathrm{P}_{7 / 2}^{\circ}$ & $a{ }^{6} D_{7 / 2}$ & $\begin{array}{c}0.0143 \\
(0.0001)\end{array}$ & $\begin{array}{c}0.0022 \\
(0.0001)\end{array}$ & $\begin{array}{c}0.0153 \\
(0.0001)\end{array}$ & $\begin{array}{c}0.0007 \\
(0.0013)\end{array}$ & $\begin{array}{l}6.0 \\
5.0 \\
4.0 \\
3.0 \\
2.0 \\
1.0 \\
2.0 \\
3.0 \\
4.0 \\
5.0 \\
6.0 \\
1.0 \\
2.0 \\
3.0 \\
4.0 \\
5.0\end{array}$ & $\begin{array}{l}5.0 \\
4.0 \\
3.0 \\
2.0 \\
1.0 \\
1.0 \\
2.0 \\
3.0 \\
4.0 \\
5.0 \\
6.0 \\
2.0 \\
3.0 \\
4.0 \\
5.0 \\
6.0 \\
\end{array}$ & $\begin{array}{l}7520.3334 \\
7520.3233 \\
7520.3129 \\
7520.3018 \\
7520.2898 \\
7520.2616 \\
7520.2593 \\
7520.2560 \\
7520.2518 \\
7520.2468 \\
7520.2413 \\
7520.2312 \\
7520.2136 \\
7520.1949 \\
7520.1753 \\
7520.1548\end{array}$ & $\begin{array}{l}13293.6473 \\
13293.6650 \\
13293.6835 \\
13293.7031 \\
13293.7243 \\
13293.7741 \\
13293.7782 \\
13293.7840 \\
13293.7915 \\
13293.8003 \\
13293.8100 \\
13293.8280 \\
13293.8590 \\
13293.8920 \\
13293.9268 \\
13293.9630\end{array}$ & $\begin{array}{l}-3.18 \\
-3.00 \\
-2.97 \\
-3.03 \\
-3.23 \\
-2.98 \\
-2.91 \\
-2.74 \\
-2.54 \\
-2.36 \\
-2.19 \\
-3.23 \\
-3.03 \\
-2.97 \\
-3.00 \\
-3.18\end{array}$ \\
\hline
\end{tabular}


R. Blackwell-Whitehead et al.: Infrared Mn I laboratory oscillator strengths for the study of late type stars and ultracool dwarfs

Table 2. continued.

\begin{tabular}{|c|c|c|c|c|c|c|c|c|c|c|}
\hline \multirow{2}{*}{$\begin{array}{l}\text { Upper } \\
\text { level }\end{array}$} & \multirow{2}{*}{$\begin{array}{c}\text { Lower } \\
\text { level }\end{array}$} & \multicolumn{4}{|c|}{ HFS Constants $\left(\mathrm{cm}^{-1}\right)^{a}$} & \multicolumn{2}{|c|}{ HFS levels } & \multicolumn{2}{|c|}{ HFS transitions ${ }^{b}$} & \multirow{2}{*}{$\begin{array}{c}\text { HFS Comp. } \\
\quad \log (g f)\end{array}$} \\
\hline & & $A_{\text {upper }}$ & $B_{\text {upper }}$ & $A_{\text {lower }}$ & $B_{\text {lower }}$ & $F_{\text {upper }}$ & $F_{\text {lower }}$ & $\sigma\left(\mathrm{cm}^{-1}\right)$ & $\lambda_{\text {air }}(\AA)$ & \\
\hline$z^{6} \mathrm{P}_{5 / 2}^{\circ}$ & $\mathrm{a}^{6} \mathrm{D}_{7 / 2}$ & $\begin{array}{c}0.0156 \\
(0.0001)\end{array}$ & $\begin{array}{l}-0.0026 \\
(0.0001)\end{array}$ & $\begin{array}{c}0.0153 \\
(0.0001)\end{array}$ & $\begin{array}{c}0.0007 \\
(0.0013)\end{array}$ & $\begin{array}{l}5.0 \\
4.0 \\
3.0 \\
2.0 \\
5.0 \\
4.0 \\
3.0 \\
2.0 \\
1.0 \\
0.0 \\
1.0 \\
2.0 \\
3.0 \\
4.0 \\
5.0\end{array}$ & $\begin{array}{l}4.0 \\
3.0 \\
2.0 \\
1.0 \\
5.0 \\
4.0 \\
3.0 \\
2.0 \\
1.0 \\
1.0 \\
2.0 \\
3.0 \\
4.0 \\
5.0 \\
6.0\end{array}$ & $\begin{array}{l}7506.1820 \\
7506.1662 \\
7506.1494 \\
7506.1325 \\
7506.1055 \\
7506.1051 \\
7506.1037 \\
7506.1020 \\
7506.1006 \\
7506.0846 \\
7506.0701 \\
7506.0562 \\
7506.0426 \\
7506.0286 \\
7506.0135\end{array}$ & $\begin{array}{l}13318.7097 \\
13318.7379 \\
13318.7676 \\
13318.7977 \\
13318.8455 \\
13318.8463 \\
13318.8488 \\
13318.8517 \\
13318.8542 \\
13318.8827 \\
13318.9083 \\
13318.9329 \\
13318.9572 \\
13318.9820 \\
13319.0088\end{array}$ & $\begin{array}{l}-4.07 \\
-3.72 \\
-3.59 \\
-3.67 \\
-2.85 \\
-2.67 \\
-2.65 \\
-2.72 \\
-2.90 \\
-2.93 \\
-2.64 \\
-2.42 \\
-2.24 \\
-2.08 \\
-1.94\end{array}$ \\
\hline$z^{4} \mathrm{P}_{5 / 2}^{\circ}$ & $a{ }^{4} D_{5 / 2}$ & $\begin{array}{l}-0.0203 \\
(0.0001)\end{array}$ & $\begin{array}{c}0.0025 \\
(0.0005)\end{array}$ & $\begin{array}{c}-0.0046 \\
(0.0001)\end{array}$ & $\begin{array}{c}0.0000 \\
(-)\end{array}$ & $\begin{array}{l}0.0 \\
1.0 \\
1.0 \\
1.0 \\
2.0 \\
2.0 \\
2.0 \\
3.0 \\
3.0 \\
3.0 \\
4.0 \\
4.0 \\
4.0 \\
5.0 \\
5.0\end{array}$ & $\begin{array}{l}1.0 \\
2.0 \\
1.0 \\
0.0 \\
3.0 \\
2.0 \\
1.0 \\
4.0 \\
3.0 \\
2.0 \\
5.0 \\
4.0 \\
3.0 \\
5.0 \\
4.0\end{array}$ & $\begin{array}{l}7452.0928 \\
7452.0813 \\
7452.0721 \\
7452.0675 \\
7452.0539 \\
7452.0401 \\
7452.0309 \\
7452.0108 \\
7451.9924 \\
7451.9786 \\
7451.9525 \\
7451.9295 \\
7451.9111 \\
7451.8520 \\
7451.8290\end{array}$ & $\begin{array}{l}13415.3805 \\
13415.4012 \\
13415.4178 \\
13415.4261 \\
13415.4506 \\
13415.4755 \\
13415.4920 \\
13415.5282 \\
13415.5613 \\
13415.5862 \\
13415.6332 \\
13415.6746 \\
13415.7077 \\
13415.8141 \\
13415.8555\end{array}$ & $\begin{array}{l}-3.29 \\
-3.02 \\
-4.05 \\
-3.29 \\
-2.92 \\
-3.35 \\
-3.02 \\
-2.93 \\
-2.91 \\
-2.92 \\
-3.09 \\
-2.58 \\
-2.93 \\
-2.31 \\
-3.09\end{array}$ \\
\hline$z^{4} \mathrm{P}_{3 / 2}^{\circ}$ & $a^{4} D_{3 / 2}$ & $\begin{array}{l}-0.0271 \\
(0.0001)\end{array}$ & $\begin{array}{l}-0.0013 \\
(0.0010)\end{array}$ & $\begin{array}{c}0.0017 \\
(0.0002)\end{array}$ & $\begin{array}{c}0.0000 \\
(-)\end{array}$ & $\begin{array}{l}1.0 \\
1.0 \\
2.0 \\
2.0 \\
2.0 \\
3.0 \\
3.0 \\
3.0 \\
4.0 \\
4.0\end{array}$ & $\begin{array}{l}1.0 \\
2.0 \\
1.0 \\
2.0 \\
3.0 \\
2.0 \\
3.0 \\
4.0 \\
3.0 \\
4.0\end{array}$ & $\begin{array}{l}7357.0508 \\
7357.0474 \\
7356.9973 \\
7356.9939 \\
7356.9888 \\
7356.9130 \\
7356.9079 \\
7356.9011 \\
7356.7988 \\
7356.7920\end{array}$ & $\begin{array}{l}13588.6873 \\
13588.6936 \\
13588.7861 \\
13588.7924 \\
13588.8018 \\
13588.9418 \\
13588.9513 \\
13588.9638 \\
13589.1528 \\
13589.1653\end{array}$ & $\begin{array}{l}-3.07 \\
-2.70 \\
-2.70 \\
-4.28 \\
-2.57 \\
-2.57 \\
-2.74 \\
-2.67 \\
-2.67 \\
-2.19\end{array}$ \\
\hline$z^{6} \mathrm{P}_{7 / 2}^{\circ}$ & $a^{6} D_{5 / 2}$ & $\begin{array}{c}0.0143 \\
(0.0001)\end{array}$ & $\begin{array}{c}0.0022 \\
(0.0001)\end{array}$ & $\begin{array}{c}0.0146 \\
(0.0001)\end{array}$ & $\begin{array}{l}-0.0016 \\
(0.0010)\end{array}$ & $\begin{array}{l}6.0 \\
5.0 \\
4.0 \\
3.0 \\
2.0 \\
1.0 \\
1.0 \\
2.0 \\
3.0 \\
4.0 \\
5.0 \\
1.0 \\
2.0 \\
3.0 \\
4.0\end{array}$ & $\begin{array}{l}5.0 \\
4.0 \\
3.0 \\
2.0 \\
1.0 \\
0.0 \\
1.0 \\
2.0 \\
3.0 \\
4.0 \\
5.0 \\
2.0 \\
3.0 \\
4.0 \\
5.0\end{array}$ & $\begin{array}{l}7350.7641 \\
7350.7499 \\
7350.7368 \\
7350.7241 \\
7350.7113 \\
7350.6980 \\
7350.6831 \\
7350.6817 \\
7350.6799 \\
7350.6783 \\
7350.6775 \\
7350.6535 \\
7350.6375 \\
7350.6215 \\
7350.6060\end{array}$ & $\begin{array}{l}13600.3091 \\
13600.3353 \\
13600.3595 \\
13600.3830 \\
13600.4067 \\
13600.4314 \\
13600.4589 \\
13600.4615 \\
13600.4648 \\
13600.4677 \\
13600.4692 \\
13600.5137 \\
13600.5433 \\
13600.5729 \\
13600.6016\end{array}$ & $\begin{array}{l}-2.90 \\
-3.04 \\
-3.20 \\
-3.38 \\
-3.60 \\
-3.89 \\
-3.86 \\
-3.68 \\
-3.61 \\
-3.64 \\
-3.81 \\
-4.63 \\
-4.56 \\
-4.68 \\
-5.03\end{array}$ \\
\hline$z^{6} \mathrm{P}_{5 / 2}^{\circ}$ & $a^{6} D_{5 / 2}$ & $\begin{array}{c}0.0156 \\
(0.0001)\end{array}$ & $\begin{array}{c}-0.0026 \\
(0.0001)\end{array}$ & $\begin{array}{c}0.0146 \\
(0.0001)\end{array}$ & $\begin{array}{l}-0.0016 \\
(0.0010)\end{array}$ & $\begin{array}{l}5.0 \\
4.0 \\
3.0 \\
2.0 \\
1.0\end{array}$ & $\begin{array}{l}4.0 \\
3.0 \\
2.0 \\
1.0 \\
0.0\end{array}$ & $\begin{array}{l}7336.6086 \\
7336.5901 \\
7336.5718 \\
7336.5539 \\
7336.5369 \\
\end{array}$ & $\begin{array}{l}13626.5500 \\
13626.5843 \\
13626.6184 \\
13626.6514 \\
13626.6831\end{array}$ & $\begin{array}{l}-2.87 \\
-2.71 \\
-2.70 \\
-2.80 \\
-3.07\end{array}$ \\
\hline
\end{tabular}


Table 2. continued.

\begin{tabular}{|c|c|c|c|c|c|c|c|c|c|c|}
\hline \multirow{2}{*}{$\begin{array}{l}\text { Upper } \\
\text { level }\end{array}$} & \multirow{2}{*}{$\begin{array}{c}\text { Lower } \\
\text { level }\end{array}$} & \multicolumn{4}{|c|}{ HFS Constants $\left(\mathrm{cm}^{-1}\right)^{a}$} & \multicolumn{2}{|c|}{ HFS levels } & \multicolumn{2}{|c|}{ HFS transitions ${ }^{b}$} & \multirow{2}{*}{$\begin{array}{l}\text { HFS Comp. }{ }^{c} \\
\quad \log (g f)\end{array}$} \\
\hline & & $A_{\text {upper }}$ & $B_{\text {upper }}$ & $A_{\text {lower }}$ & $B_{\text {lower }}$ & $F_{\text {upper }}$ & $F_{\text {lower }}$ & $\sigma\left(\mathrm{cm}^{-1}\right)$ & $\lambda_{\text {air }}(\AA)$ & \\
\hline & & & & & & 5.0 & 5.0 & 7336.5362 & 13626.6844 & -2.09 \\
\hline & & & & & & 4.0 & 4.0 & 7336.5316 & 13626.6929 & -2.36 \\
\hline & & & & & & 3.0 & 3.0 & 7336.5276 & 13626.7004 & -2.69 \\
\hline & & & & & & 2.0 & 2.0 & 7336.5243 & 13626.7065 & -3.13 \\
\hline & & & & & & 1.0 & 1.0 & 7336.5221 & 13626.7107 & -3.83 \\
\hline & & & & & & 0.0 & 1.0 & 7336.5061 & 13626.7404 & -3.07 \\
\hline & & & & & & 1.0 & 2.0 & 7336.4925 & 13626.7657 & -2.80 \\
\hline & & & & & & 2.0 & 3.0 & 7336.4801 & 13626.7885 & -2.70 \\
\hline & & & & & & 3.0 & 4.0 & 7336.4691 & 13626.8090 & -2.71 \\
\hline & & & & & & 4.0 & 5.0 & 7336.4593 & 13626.8273 & -2.87 \\
\hline \multirow[t]{12}{*}{$z^{6} \mathrm{P}_{3 / 2}^{\circ}$} & $\mathrm{a}^{6} \mathrm{D}_{5 / 2}$ & 0.0191 & 0.0006 & 0.0146 & -0.0016 & 4.0 & 3.0 & 7327.9113 & 13642.7230 & -3.98 \\
\hline & & $(0.0001)$ & $(0.0002)$ & $(0.0001)$ & $(0.0010)$ & 3.0 & 2.0 & 7327.8787 & 13642.7836 & -3.60 \\
\hline & & & & & & 4.0 & 4.0 & 7327.8528 & 13642.8319 & -3.02 \\
\hline & & & & & & 2.0 & 1.0 & 7327.8512 & 13642.8348 & -3.42 \\
\hline & & & & & & 3.0 & 3.0 & 7327.8345 & 13642.8659 & -2.88 \\
\hline & & & & & & 1.0 & 0.0 & 7327.8282 & 13642.8777 & -3.38 \\
\hline & & & & & & 2.0 & 2.0 & 7327.8216 & 13642.8900 & -2.90 \\
\hline & & & & & & 1.0 & 1.0 & 7327.8133 & 13642.9053 & -3.05 \\
\hline & & & & & & 1.0 & 2.0 & 7327.7837 & 13642.9605 & -3.23 \\
\hline & & & & & & 4.0 & 5.0 & 7327.7804 & 13642.9666 & -2.33 \\
\hline & & & & & & 2.0 & 3.0 & 7327.7774 & 13642.9722 & -2.82 \\
\hline & & & & & & 3.0 & 4.0 & 7327.7761 & 13642.9747 & -2.55 \\
\hline \multirow{12}{*}{$z^{4} \mathrm{P}_{5 / 2}^{\circ}$} & $\mathrm{a}^{4} \mathrm{D}_{3 / 2}$ & -0.0203 & 0.0025 & 0.0017 & 0.0000 & 0.0 & 1.0 & 7281.8174 & 13729.0815 & -4.21 \\
\hline & & $(0.0001)$ & $(0.0005)$ & $(0.0002)$ & $(-)$ & 1.0 & 1.0 & 7281.7967 & 13729.1206 & -3.88 \\
\hline & & & & & & 1.0 & 2.0 & 7281.7933 & 13729.1270 & -4.25 \\
\hline & & & & & & 2.0 & 1.0 & 7281.7554 & 13729.1983 & -4.06 \\
\hline & & & & & & 2.0 & 2.0 & 7281.7520 & 13729.2047 & -3.72 \\
\hline & & & & & & 2.0 & 3.0 & 7281.7469 & 13729.2144 & -4.43 \\
\hline & & & & & & 3.0 & 2.0 & 7281.6905 & 13729.3207 & -3.65 \\
\hline & & & & & & 3.0 & 3.0 & 7281.6854 & 13729.3303 & -3.71 \\
\hline & & & & & & 3.0 & 4.0 & 7281.6786 & 13729.3431 & -4.81 \\
\hline & & & & & & 4.0 & 3.0 & 7281.6041 & 13729.4836 & -3.38 \\
\hline & & & & & & 4.0 & 4.0 & 7281.5973 & 13729.4964 & -3.85 \\
\hline & & & & & & 5.0 & 4.0 & 7281.4968 & 13729.6859 & -3.16 \\
\hline \multirow[t]{6}{*}{$z^{4} \mathrm{P}_{3 / 2}^{\circ}$} & $a{ }^{4} D_{1 / 2}$ & -0.0271 & -0.0013 & 0.0506 & 0.0000 & 1.0 & 2.0 & 7257.7805 & 13774.5508 & -3.35 \\
\hline & & $(0.0001)$ & $(0.0010)$ & $(0.0003)$ & $(-)$ & 2.0 & 2.0 & 7257.7269 & 13774.6523 & -3.24 \\
\hline & & & & & & 3.0 & 2.0 & 7257.6460 & 13774.8059 & -3.34 \\
\hline & & & & & & 2.0 & 3.0 & 7257.5751 & 13774.9404 & -3.78 \\
\hline & & & & & & 3.0 & 3.0 & 7257.4942 & 13775.0940 & -3.24 \\
\hline & & & & & & 4.0 & 3.0 & 7257.3851 & 13775.3011 & -2.88 \\
\hline \multirow[t]{12}{*}{$z^{6} \mathrm{P}_{5 / 2}^{\circ}$} & $a{ }^{6} D_{3 / 2}$ & 0.0156 & -0.0026 & 0.0157 & -0.0022 & 5.0 & 4.0 & 7219.6085 & 13847.3805 & -2.49 \\
\hline & & $(0.0001)$ & $(0.0001)$ & $(0.0002)$ & $(0.0017)$ & 4.0 & 3.0 & 7219.5932 & 13847.4099 & -2.71 \\
\hline & & & & & & 3.0 & 2.0 & 7219.5784 & 13847.4382 & -2.98 \\
\hline & & & & & & 2.0 & 1.0 & 7219.5636 & 13847.4667 & -3.39 \\
\hline & & & & & & 1.0 & 1.0 & 7219.5317 & 13847.5279 & -3.21 \\
\hline & & & & & & 4.0 & 4.0 & 7219.5315 & 13847.5282 & -3.18 \\
\hline & & & & & & 2.0 & 2.0 & 7219.5310 & 13847.5292 & -3.06 \\
\hline & & & & & & 3.0 & 3.0 & 7219.5307 & 13847.5298 & -3.04 \\
\hline & & & & & & 0.0 & 1.0 & 7219.5157 & 13847.5586 & -3.54 \\
\hline & & & & & & 1.0 & 2.0 & 7219.4991 & 13847.5903 & -3.58 \\
\hline & & & & & & 2.0 & 3.0 & 7219.4832 & 13847.6208 & -3.76 \\
\hline & & & & & & 3.0 & 4.0 & 7219.4690 & 13847.6480 & -4.14 \\
\hline \multirow[t]{8}{*}{$z^{6} \mathrm{P}_{3 / 2}^{\circ}$} & $a{ }^{6} D_{3 / 2}$ & 0.0191 & 0.0006 & 0.0157 & -0.0022 & 4.0 & 3.0 & 7210.9541 & 13863.9998 & -3.81 \\
\hline & & $(0.0001)$ & $(0.0002)$ & $(0.0002)$ & $(0.0017)$ & 3.0 & 2.0 & 7210.9250 & 13864.0558 & -3.43 \\
\hline & & & & & & 2.0 & 1.0 & 7210.8998 & 13864.1041 & -3.25 \\
\hline & & & & & & 4.0 & 4.0 & 7210.8912 & 13864.1208 & -2.85 \\
\hline & & & & & & 1.0 & 0.0 & 7210.8780 & 13864.1461 & -3.21 \\
\hline & & & & & & 3.0 & 3.0 & 7210.8774 & 13864.1473 & -2.71 \\
\hline & & & & & & 2.0 & 2.0 & 7210.8679 & 13864.1656 & -2.73 \\
\hline & & & & & & 1.0 & 1.0 & 7210.8620 & 13864.1770 & -2.88 \\
\hline
\end{tabular}


R. Blackwell-Whitehead et al.: Infrared Mn I laboratory oscillator strengths for the study of late type stars and ultracool dwarfs

Table 2. continued.

\begin{tabular}{|c|c|c|c|c|c|c|c|c|c|c|}
\hline \multirow{2}{*}{$\begin{array}{l}\text { Upper } \\
\text { level }\end{array}$} & \multirow{2}{*}{$\begin{array}{c}\text { Lower } \\
\text { level }\end{array}$} & \multicolumn{4}{|c|}{ HFS Constants $\left(\mathrm{cm}^{-1}\right)^{a}$} & \multicolumn{2}{|c|}{ HFS levels } & \multicolumn{2}{|c|}{ HFS transitions $^{b}$} & \multirow{2}{*}{$\begin{array}{c}\text { HFS Comp. } \\
\quad \log (g f)\end{array}$} \\
\hline & & $A_{\text {upper }}$ & $B_{\text {upper }}$ & $A_{\text {lower }}$ & $B_{\text {lower }}$ & $F_{\text {upper }}$ & $F_{\text {lower }}$ & $\sigma\left(\mathrm{cm}^{-1}\right)$ & $\lambda_{\text {air }}(\AA)$ & \\
\hline & & & & & & 1.0 & 2.0 & 7210.8300 & 13864.2384 & -3.06 \\
\hline & & & & & & 2.0 & 3.0 & 7210.8202 & 13864.2572 & -2.65 \\
\hline & & & & & & 3.0 & 4.0 & 7210.8145 & 13864.2683 & -2.38 \\
\hline & & & & & & 4.0 & 5.0 & 7210.8136 & 13864.2700 & -2.16 \\
\hline \multirow[t]{6}{*}{$z^{6} \mathrm{P}_{3 / 2}^{\circ}$} & $a^{6} D_{1 / 2}$ & 0.0191 & 0.0006 & 0.0294 & 0.0000 & 3.0 & 2.0 & 7142.1563 & 13997.5468 & -2.67 \\
\hline & & $(0.0001)$ & $(0.0002)$ & $(0.0004)$ & $(-)$ & 4.0 & 3.0 & 7142.1449 & 13997.5693 & -2.21 \\
\hline & & & & & & 2.0 & 2.0 & 7142.0992 & 13997.6588 & -2.57 \\
\hline & & & & & & 3.0 & 3.0 & 7142.0681 & 13997.7197 & -2.57 \\
\hline & & & & & & 1.0 & 2.0 & 7142.0613 & 13997.7330 & -2.68 \\
\hline & & & & & & 2.0 & 3.0 & 7142.0110 & 13997.8316 & -3.11 \\
\hline
\end{tabular}

Notes. ${ }^{(a)}$ The HFS $A$ and $B$ constants are from the published values of Dembczyński et al. (1979) for the a ${ }^{6} \mathrm{D}_{J}$ levels, Handrich et al. (1969) for the $z^{6} \mathrm{P}_{J}^{\circ}$ levels, Blackwell-Whitehead et al. (2005b) for the a ${ }^{4} \mathrm{D}_{J}$ levels and Brodzinski et al. (1987) for the $z^{4} \mathrm{P}_{J}^{\circ}$ levels. Where the $B$ constant is quoted as $0.0000(-)$ the $B$ constant was fixed at zero in the cited reference. The uncertainty in parentheses is taken from the aforementioned references. ${ }^{(b)}$ The wavenumber and wavelength positions for the HFS transitions have been determined from energy levels reported in the NIST atomic Spectra Database (Ralchenko et al. 2009). The relative uncertainty in the wavenumber and wavelength of the HFS transitions is determined from the uncertainty in the HFS constants for the upper and lower fine structure levels, $\mathrm{d} \sigma_{\max }=0.001 \mathrm{~cm}^{-1}$ and $\mathrm{d} \lambda_{\max }=0.002 \AA$. However, the wavenumber and wavelength in the table are given to four decimal places to adequately identify the hyperfine component lines. ${ }^{(c)}$ The $\log (g f)$ for the HFS component lines is determined from the hyperfine splitting constants and $\log (g f)$ for the complete fine structure transition profile.
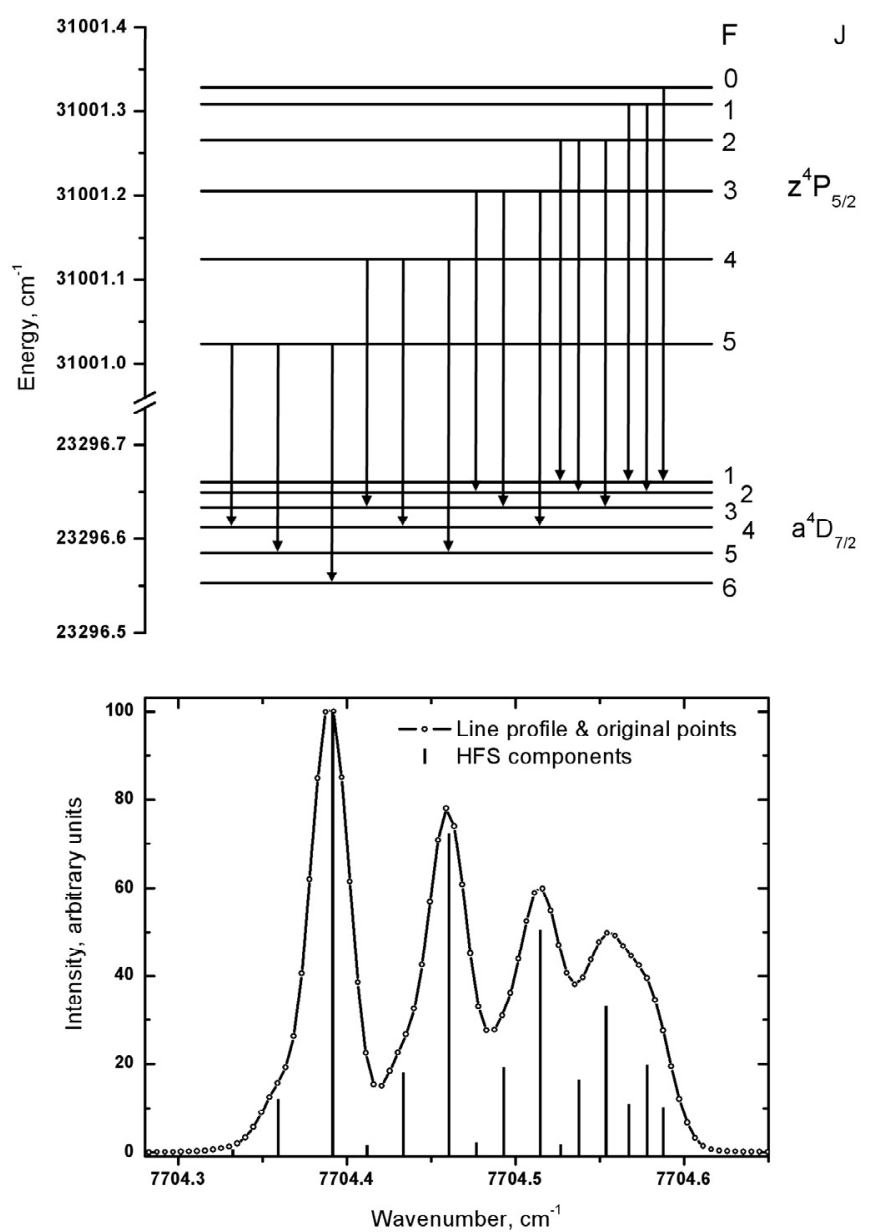

Fig. 1. The upper plot shows the hyperfine splitting of the fine structure levels $3 \mathrm{~d}^{6}\left({ }^{5} \mathrm{D}\right) 4 \mathrm{~s}$ a ${ }^{4} \mathrm{D}_{7 / 2}-3 \mathrm{~d}^{5}\left({ }^{6} \mathrm{~S}\right) 4 \mathrm{~s} 4 \mathrm{p}\left({ }^{3} \mathrm{P}\right) z^{4} \mathrm{P}_{5 / 2}^{\circ}$ with allowed hyperfine transitions. The lower plot shows the hyperfine split profile of the transition observed in the uncalibrated laboratory spectrum at $12975 \AA$, together with an indication of the positions and relative line strengths of the individual HFS transitions. A complete list of the Ritz wavelength and $\log (g f)$ for each HFS transition is available in Table 2.

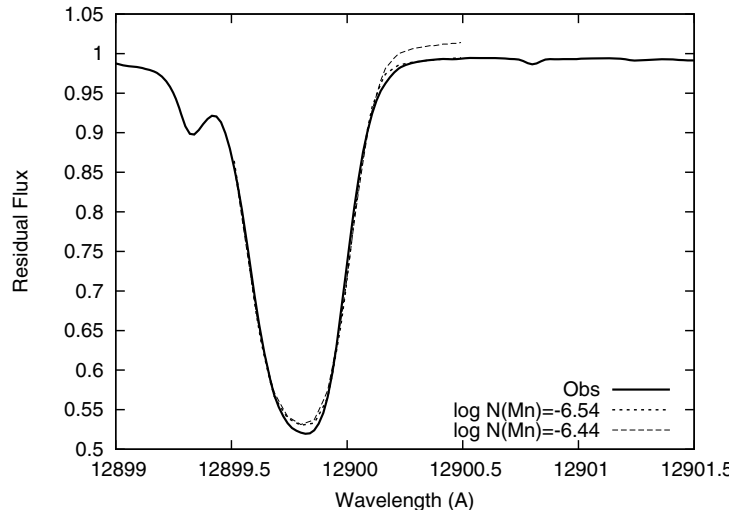

Fig. 2. The best fit to the observed solar spectrum feature found from the minima of Eq. (3) for the Mn I line at $12899 \AA$.

the instrumental broadening. For Arcturus, a rotational broadening was added corresponding to a projected equatorial radial velocity $v \sin i=7 \mathrm{~km} \mathrm{~s}^{-1}$, where $v$ is the equatorial velocity and $i$ is the inclination of the stellar rotation axis to the line of sight to the Earth, by following the Gray (1976) formulae.

We have fitted our synthetic spectra to the observed spectra of the Sun and Arcturus atlases by Kurucz (1991) and Hinkle et al. (1995) respectively. To obtain the best fit to the observed spectra we followed the minimisation procedure described by Pavlenko \& Jones (2002) and Jones et al. (2002). In summary, we find the minima of the $3 \mathrm{D}$ function

$S\left(f_{\mathrm{s}}, f_{\mathrm{h}}, f_{\mathrm{g}}\right)=\sum_{v}\left(F_{v}-F_{v}^{x}\right)^{2}$,

where $F_{v}$ and $F_{v}^{x}$ are the observed and computed spectra respectively, and $f_{\mathrm{s}}, f_{\mathrm{h}}, f_{\mathrm{g}}$ are the wavelength shift, the normalisation factor, and the profile broadening parameter, respectively. To estimate the uncertainty of the best fit we use the parameter $\Delta S=\sqrt{\frac{S}{N(N-1)}}$, where $N$ is the number of points in the observed spectrum. We provide an example of the profile fit for the Mn I at $12899 \AA$ line in the spectrum of the Sun in Fig. 2 and the 

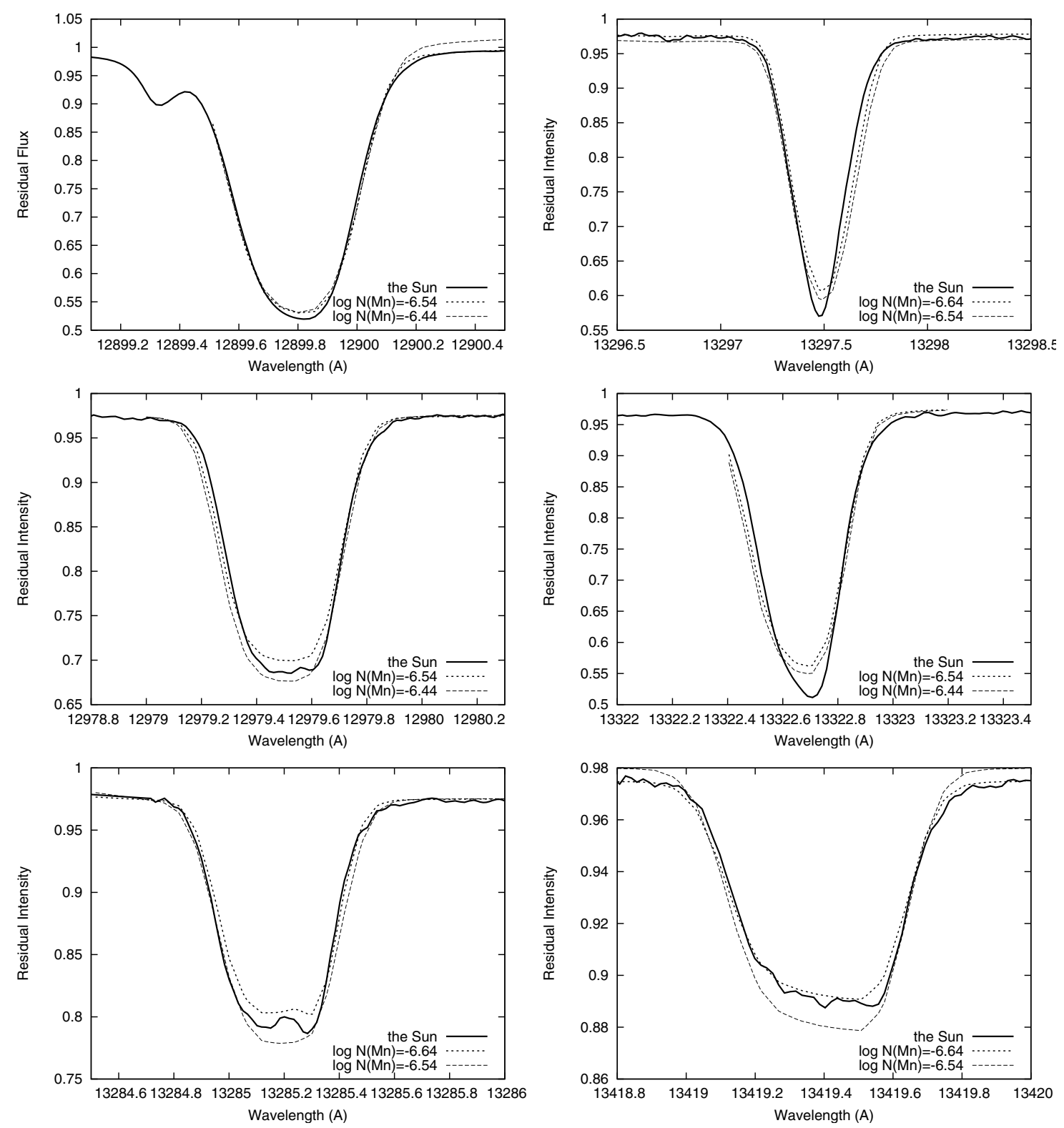

Fig. 3. The best fits to the observed solar spectrum features found from the minima of Eq. (3) for Mn I lines at $12899 \AA, 12975 \AA$, $13281 \AA$ (left column) and $13293 \AA, 13318 \AA$, $13415 \AA$ (right column). The wavelength scale is taken from the observed spectrum of the Sun.

fitted profiles of the other IR transitions $(12975,13281,13293$, 13318 and $13415 \AA$ ) are shown in Fig. 3 .

\subsection{MnI lines in spectra of the Sun}

Fits of our synthetic spectra to the observed spectrum of the Sun are shown in Fig. 3. The manganese abundances obtained from the fits are given in Table 3 . The $\log N(\mathrm{Mn})$ values in Table 3 are determined from a grid of manganese abundances, using the solar value $\log N(\mathrm{Mn})=-6.64$ of Gurtovenko \& Kostik (1989) and 0.05 and 0.1 dex as the abundance steps for the $\log N(\mathrm{Mn})$ measurements.

\subsection{Mn I lines in spectra of Arcturus}

Manganese lines in the Arcturus spectrum are considerably more blended. Our fits to the observed profiles of Mn I lines are shown in Fig. 4, and the results of manganese abundance determination
Table 3. The Mn I abundances determined from the best fits to the solar spectrum.

\begin{tabular}{lc}
\hline \hline Mn I line (approx. Å) & $\log N(\mathrm{Mn})$ \\
\hline 12899 & -6.54 \\
12975 & -6.54 \\
13281 & -6.64 \\
13293 & -6.64 \\
13318 & -6.54 \\
13415 & -6.54 \\
\hline
\end{tabular}

Notes. The uncertainty in the $\log N(\mathrm{Mn})$ values is \pm 0.05 and relates to the step size in the grid of manganese abundances.

are shown in Table 4. The increase in the dispersion of the abundance results for Arcturus in comparison to the Sun is predominantly caused by the increase in blended features in the Arcturus spectrum. To minimise the effect of blending we exclude spectral regions with a relatively high number of unidentified blended 
R. Blackwell-Whitehead et al.: Infrared Mn I laboratory oscillator strengths for the study of late type stars and ultracool dwarfs
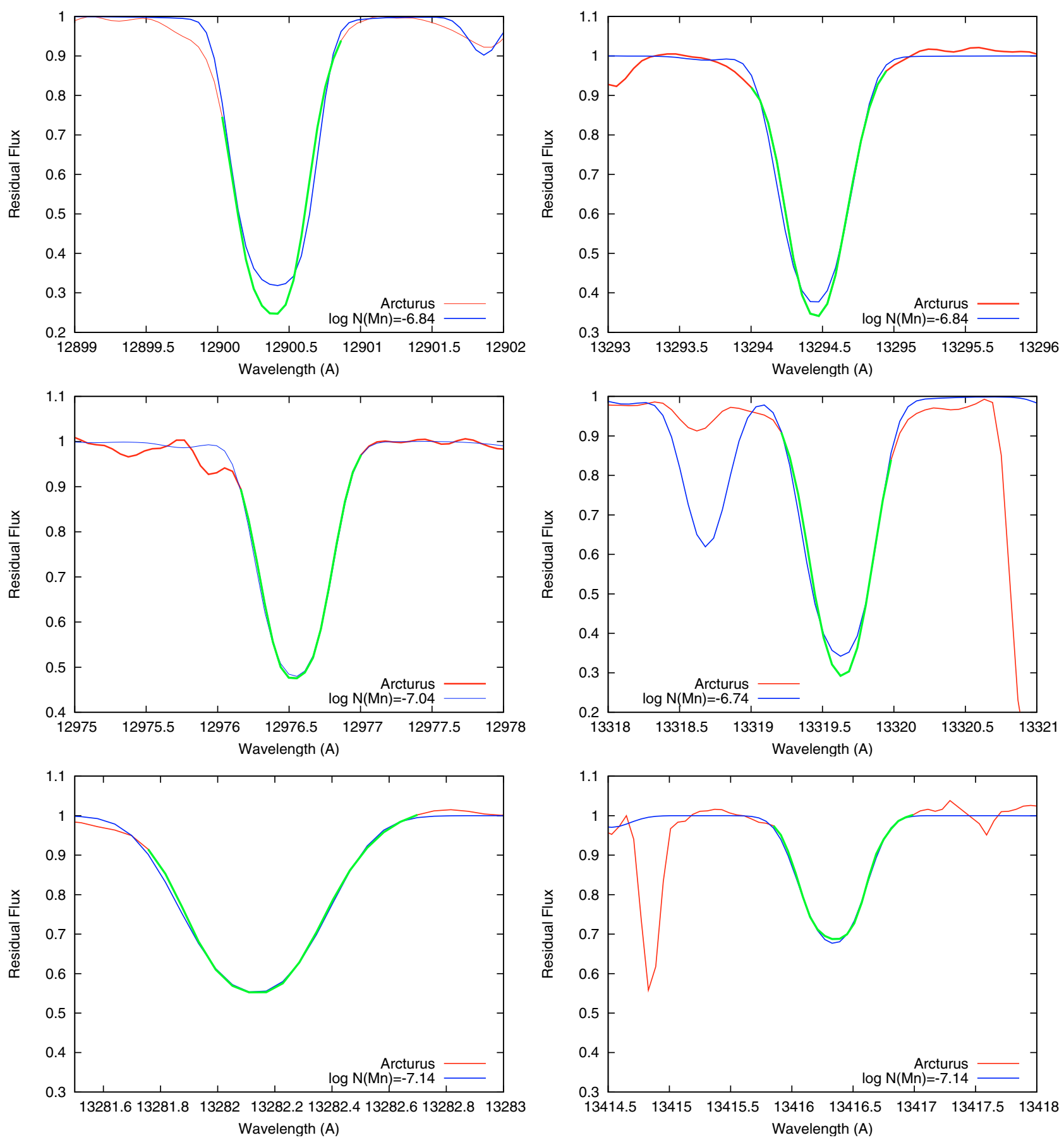

Fig. 4. The best fits to the observed Arcturus spectrum features found from the minima of Eq. (3) for Mn I lines at $12899 \AA, 12975 \AA$, $13281 \AA$ (left column) and $13293 \AA, 13318 \AA, 13415 \AA$ (right column). The green line shows the section of the observed profile used to determine manganese abundance. The wavelength scale is taken from the observed spectrum of Arcturus.

Table 4. The MnI abundances determined from the best fits to the Arcturus spectrum.

\begin{tabular}{lc}
\hline \hline Mn I line (fit range, $\AA$ ) & $\log N(\mathrm{Mn})$ \\
\hline $12899.8-12900.9$ & -6.9 \\
$12976.15-12999.0$ & -7.1 \\
$13281.7-13282.7$ & -7.2 \\
$13294.0-13295.0$ & -6.9 \\
$13319.2-13320.0$ & -6.8 \\
$13415.8-13417.0$ & -7.2 \\
\hline
\end{tabular}

Notes. The uncertainty in the $\log N(\mathrm{Mn})$ values is 0.1 and relates to the step size in the grid of manganese abundances. features when fitting our calculated spectra to the observed spectrum. The corresponding wavelength range for each line is shown in Table 4 and the unblended spectral region used for our fit is marked by a green line in Fig. 4. In addition, in Fig. 5 we provide plots of the dependance of $S$ with $\log N(\mathrm{Mn})$ for the Mn I lines (12899 ̊, $12975 \AA, 13281 \AA, 13293 \AA$, $13318 \AA$, $13415 \AA$ ) observed in the spectrum of Arcturus.

\section{3. $\mathrm{MnI}$ lines in spectra of ultracool dwarfs}

The blending of Mn I lines in the IR spectrum of late-type objects increases significantly for effective temperatures lower than 

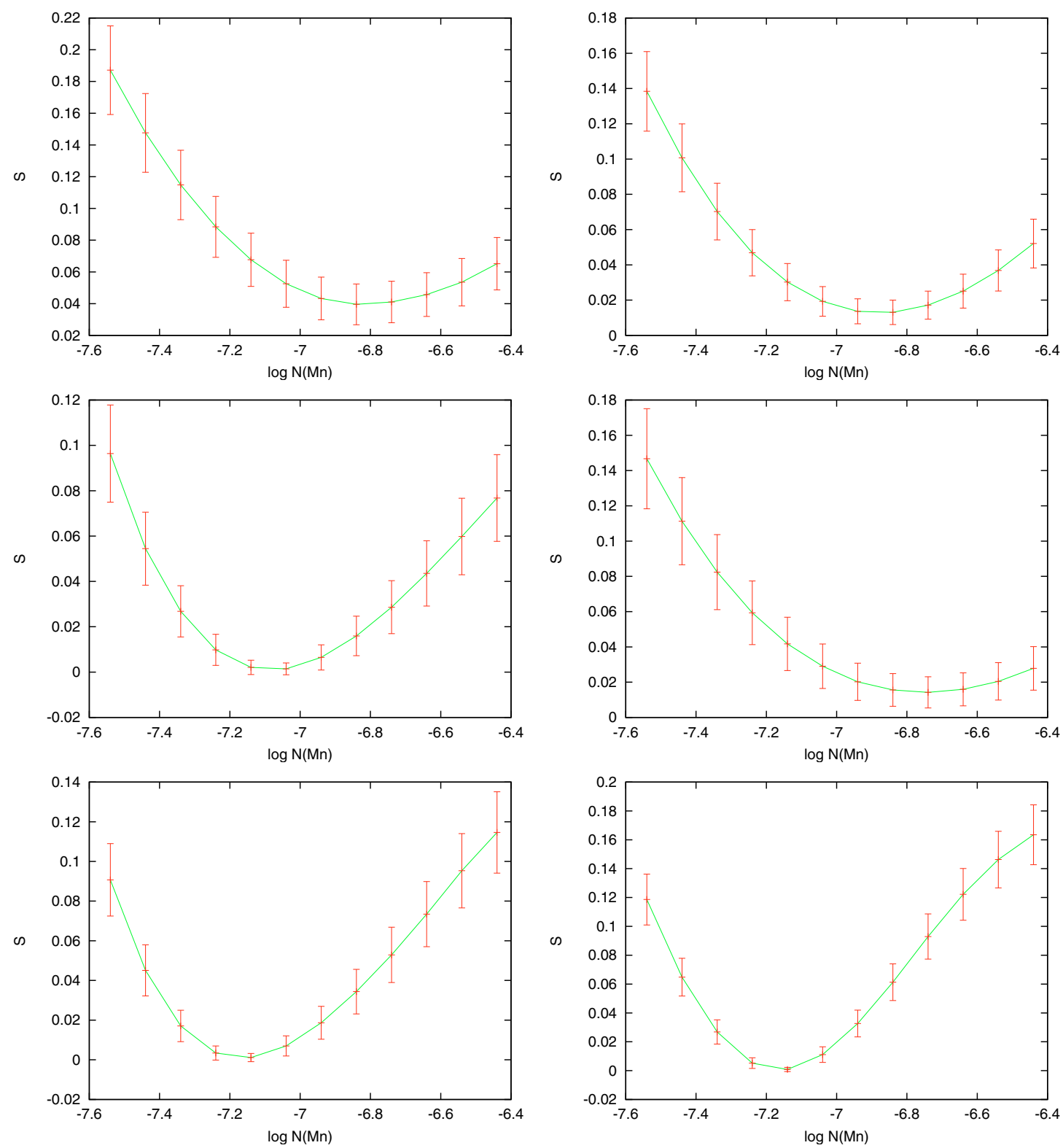

Fig. 5. Dependence of $S$ on $\log N(\mathrm{Mn})$ for the Mn I lines at $12899 \AA$, $12975 \AA$, $13281 \AA$ (left column, top to bottom) and $13293 \AA$, $13318 \AA$, $13415 \AA$ (right column, top to bottom) observed in spectrum of Arcturus, see Fig. 4.

3000 K. Numerous water lines form a pseudo background in the spectral region of many of the IR Mn I lines. However, for M9 dwarfs it is possible to fit the observed profiles of some Mn I lines. We provide an example of the profile fit for the Mn I line at $12899 \AA$ in the spectrum of 2MASSW $0140026+270150$. The observed spectrum is described in detail in Lyubchik et al. (2007). The computation was performed with an initial assumption of solar like abundances for manganese, and other elements, and we have determined a value of $\log N(\mathrm{Mn})=-6.7 \pm 0.2$ in the atmosphere of 2MASSW 0140026+270150, which agrees to within the joint uncertainties with our derived solar abundance for manganese.

Both the line intensity and line profile can be fitted with the solar value of the manganese abundance. However, the spectra of cooler objects are dominated by the water bands in the near-IR, see the computed spectrum of LP944-20 (M 9.5, 2000/4.5 from Pavlenko et al. 2007). For these cooler objects only the Mn I line at $12899 \AA$ can be used for the analysis because the other lines are too blended with the $\mathrm{H}_{2} \mathrm{O}$ bands.

\section{Summary}

Branching fractions for $20 \mathrm{Mn}$ I transitions have been measured using high resolution Fourier transform spectroscopy and placed on an absolute scale using radiative lifetimes. Fifteen of these transitions have no previously published experimentally measured oscillator strengths. The remaining five transitions agree with previous published oscillator strengths to within the uncertainty of the measurements.

Using our new experimental $\log (g f)$ values we have determined the manganese abundance in the atmosphere of several late type stars. Our solar manganese abundance, $\log N(\mathrm{Mn})=$ $-6.60 \pm 0.05$, agrees well with the manganese abundances of Anders \& Grevesse (1989) $\log N(\mathrm{Mn})=-6.65$, Gurtovenko $\&$ Kostik (1989) $\log N(\mathrm{Mn})=-6.64$, and Biemont (1975) $\log N(\mathrm{Mn})=6.67$. Blending affects $\mathrm{Mn}$ I lines in the IR spectra of stars cooler than the Sun. As a result, our manganese abundances obtained from the fits to different lines in the spectrum of Arcturus are in the range $-6.75<\log N(\mathrm{Mn})<-7.15$, with a 

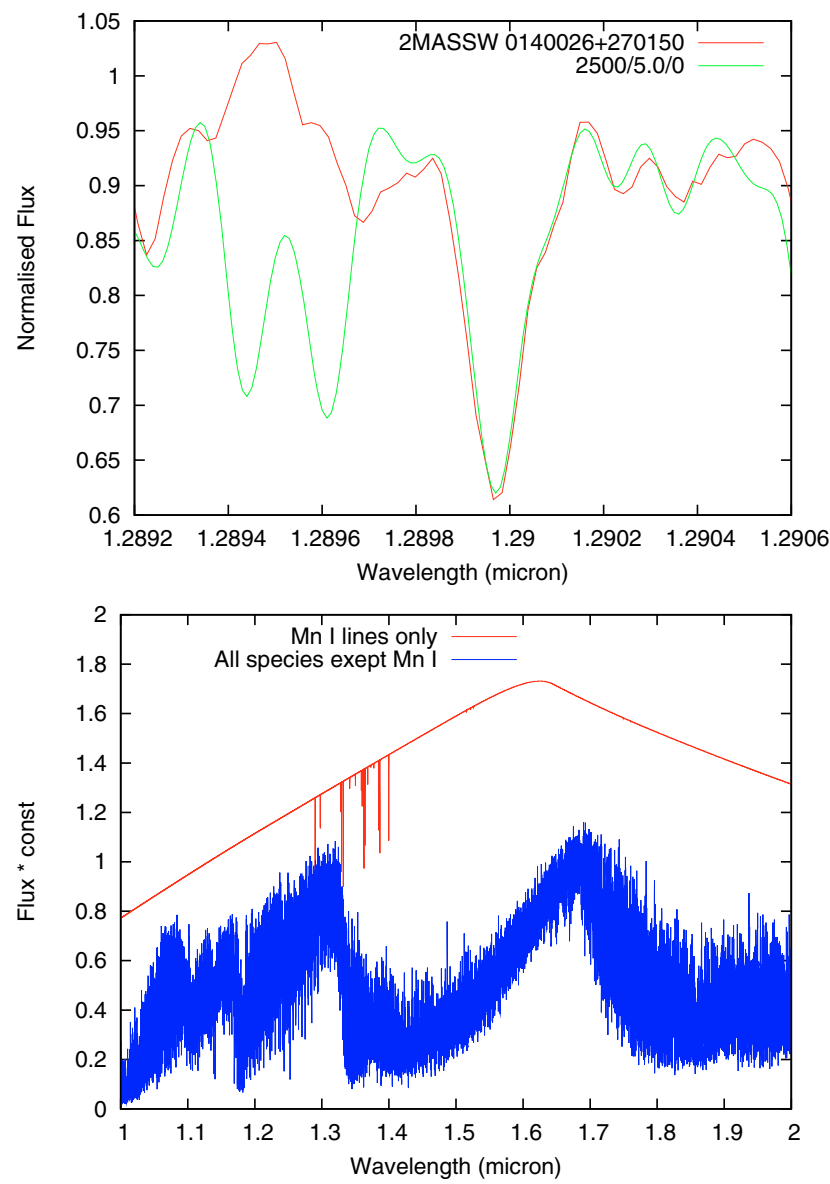

Fig. 6. Top: the manganese line at $12899 \AA$ in the spectrum of M 9.5 dwarf 2MASSW $0140026+270150$, where the red line is the observed spectrum and the green line is the model spectrum using our oscillator strengths and hyperfine component line positions $\left(T_{\text {eff }} / \log (g) /[\mathrm{Fe} / \mathrm{H}]=2500 / 5.0 / 0.0\right)$. Bottom: a comparison of the calculated spectrum of LP944-20 with only absorption features from Mn I in red and all other atomic and molecular species including the water vapour bands in blue.

mean $\log N(\mathrm{Mn})=-6.95 \pm 0.20$, which agrees to within the uncertainty with the $\log N(\mathrm{Mn})=-6.97$ determined by McWilliam et al. (2003) for Arcturus. Furthermore, our new laboratory measured $\log (g f)$ s for IR Mn I spectral lines can be used in the study of dust obscured objects and as a secondary abundance check to abundance studies using Mn I spectral lines in the visible.

Acknowledgements. We thank Drs. L. Prato and I. S. McLean for providing the 2MASSW $0140026+270150$ spectrum. R.B.W. gratefully acknowledges the European Commission for a Marie Curie fellowship. J.C.P., Y.P. and H.R.A.J. gratefully acknowledge funding from the Leverhulme Trust and STFC, UK. The work of Y.P. and L.Y. was partially supported by the Microcosmophysics-2 program of the National Academy and Space Agency of Ukraine. H.N. acknowledges the support of the Linnaeus grant to the Lund Laser Centre from the Swedish Research Council.

\section{References}

Allard, F., Hauschildt, P. H., Alexander, D. R., Tamanai, A., \& Schweitzer, A. 2001, ApJ, 556, 357

Anders, E., \& Grevesse, N. 1989, Geochim Cosmochim. Acta, 53, 197

Barber, R. J., Tennyson, J., Harris, G. J., \& Tolchenov, R. 2006, MNRAS, 368, 1087

Biemont, E. 1975, Sol. Phys., 44, 269

Bigot, L., \& Thévenin, F. 2006, MNRAS, 372, 609
Birch, K. P., \& Downs, M. J. 1994, Metrologia, 31, 315

Blackwell-Whitehead, R. J., Xu, H. L., Pickering, J. C., Nave, G., \& Lundberg, H. 2005a, MNRAS, 361, 1281

Blackwell-Whitehead, R. J., Pickering, J. C., Pearse, O., \& Nave, G. 2005b, ApJS, 157, 402

Blackwell-Whitehead, R. J., Pickering, J. C., Jones, H. R. A., et al. 2008, J. Phys.: Conf. Ser., 130, 012002

Bond, J. C., Lauretta, D. S., Tinney, C. G., et al. 2008, ApJ, 682, 1234

Booth, A. J., Blackwell, D. E., Petford, A. D., \& Shallis, M. J. 1984, MNRAS, 208, 147

Brickhouse, N., Federman, S., Kwong, V., et al. 2006, Laboratory Astrophysics White Paper, Proceedings of the NASA Lab. Astro. Workshop, http:// adsabs. harvard. edu/abs/2006nla . . conf.....1B

Brodzinski, T., Kronfeldt, H. D., Kropp, J. R., \& Winkler, R. 1987, Z. Phys. D, 7,161

Burrows, A., Ram, R. S., Bernath, P., Sharp, C. M., \& Milson, J. A. 2002, ApJ, 577,986

Catalán, M. A., Meggers, W. F., \& Garcia-Riquelme, O. 1964, J. Res. Natl. Bur. Stand., 68A, 9

Dembczyński, J., Ertmer, W., Johann, U., Penselin, S., \& Stinner, P. 1979, Z. Phys. A, 291, 207

Dulick, M., Bauschlicher, C. W., Jr., Burrows, A., et al. 2003, ApJ, 594, 651

Edlén, B. 1966, Metrologia, 2, 71

Fuhr, J. R., \& Wiese W. L. 2003, NIST Atomic Transition Probability Tables, in Handbook of Chemistry and Physics, 84th edition, ed. D. R. Lide (Boca Raton, FL: CRC Press), 10, 93

Gray, D. F. 1976, The observation and analysis of stellar photospheres (New York: Wiley-Interscience), 484

Gurtovenko, E. A., \& Kostyk, R. I. 1989, Fraungoferov spektr i sistema solnechnykh sil ostsilliatorov (Kiev: Nauk. Dumka)

Handrich, E., Steudel, A., \& Walther, H. 1969, Phys. Lett., 29A, 486

Hinkle, K., Wallace, L., \& Livingston, W. 1995, PASP, 107, 1402

Johansson, S. 2005, Atomic Data in the 1-5 Micron Region: Present Status in High Resolution Infrared Spectroscopy in Astronomy (Berlin: Springer), 62

Jones, H. R. A., Pavlenko, Y. V., Tennyson, J., \& Viti, S. 2002, MNRAS, 330, 675

Jones, H. R. A., Viti S., Tennyson, J., et al. 2005, Astron. Nachr., 326, 920

Kronfeldt, H. D., Kropp, J. R., Subaric, A., \& Winkler, R. 1985, Z. Phys., 322, 349

Kuhn, H. G. 1964, Atomic Spectra (London: Longmans), 329

Kupka, F., Piskunov, N. E., Ryabchikova, T. A., Stempels, H. C., \& Weiss, W. W. 1999, A\&AS 138, 119

Kurucz, R. L. 1991, The solar spectrum, Solar interior and atmosphere (A9236201 14-92) (Tucson, AZ: University of Arizona Press), 663

Kurucz, R.l. 1993, A new opacity-sampling model atmosphere program for arbitary abundances, Peculiar versus normal phenomena in A-type and related stars, ed. M. M. Dworetsky, F. Castelli, \& R. Faragianna, ASP Conf. Ser., 44, 87

Kurucz, R. L., \& Bell, B. 1995, Atomic Line Data, Kurucz CD-ROM No. 23. Cambridge, Mass.: Smithsonian Astrophysical Observatory

Lyubchik, Y., Jones, H. R. A., Pavlenko, Y. V., et al. 2004, A\&A, 416, 655

Lyubchik, Y., Jones, H. R. A., Pavlenko, Y. V., et al. 2007, A\&A, 473, 257

Meléndez, J. 1999, MNRAS, 307, 197

Nave, G., Sansonetti, C. J., \& Griesmann, U. 1997, in Opt. Soc. Am. Tech. Digest Series, 3, Fourier transform spectroscopy: Methods and Applications (Washington DC: Opt. Soc. Am.), 38

McWilliam, A., Rich, R. M., \& Smecker-Hane, T. A. 2003, ApJ, 592, 21

Pavlenko, Ya. V. 2000, Astron. Rep., 44, 219

Pavlenko, Ya. V. 2003, Astron. Rep., 47, 59

Pavlenko, Ya. V., \& Jones, H. R. A. 2002, A\&A, 396, 967

Pavlenko, Ya. V., Jones, H. R. A., Martín, E. L., et al. 2007, MNRAS, 380, 1285

Peterson, R., Ore, C. M. D., \& Kurucz, R. 1993, ApJ, 404, 333

Pickering J. C. 2002, Vib. Spectrosc., 29, 27

Plez, B. 1998, A\&A, 337, 495

Prochaska, J. X., \& McWilliam, A. 2000, ApJ, 537, L37

Ralchenko, Yu., Kramida, A. E., Reader, J., \& NIST ASD Team 2008, NIST Atomic Spectra Database (version 3.1.5), http://physics.nist.gov/ asd3 [2009, June 18], National Institute of Standards and Technology, Gaithersburg, MD

Schnabel, R., Bard, A., \& Kock, M. 1995, Z. Phys. D, 34, 223

Sikström, C. M., Nilsson, H., Litzén, U., Blom, A., \& Lundberg, H. 2002, J. Quant. Spec. Rad. Trans., 74, 355

Sobeck, J. S., Ivans, I. I., Simmerer, J. A., et al. 2006, AJ, 131, 2949

Taylor, B. N., \& Kuyatt, C. E. 1994, Guidelines for Evaluating and Expressing the Uncertainty of NIST Measurement Results, NIST Technical Note, 1297

Unsöld, A. 1955, Physik der Sternatmospheren, 2nd edn. (Berlin: Springer)

Wahlgren, G. M., \& Johansson, S. 2003, GAIA Spectroscopy: Science and Technology, ASP Conf. Proc., ed. U. Munari, 298, 481 